\title{
Speckle Noise Reduction in Michelson Digital Holography Using Known or Unknown Reference Linear Phases and Image Processing
}

\author{
Tomi Pitkäaho $^{1}$ and Thomas J. Naughton ${ }^{2}$ \\ ${ }^{1}$ University of Oulu, RFMedia Laboratory, \\ Oulu Southern Institute, \\ Vierimaantie 5, 84100 Ylivieska, Finland \\ tomi.pitkaaho@oulu.fi \\ ${ }^{2}$ Department of Computer Science, \\ National University of Ireland Maynooth, \\ Maynooth, County Kildare, Ireland \\ tomn@cs.nuim.ie
}

\section{Introduction}

Speckle is caused by the illumination of an optically rough surface by coherent light [1] which can cause problems in digital holography. Speckle in a reconstruction reduces visibility of details and may impede important measurements of an object. Speckle can be reduced by capturing multiple holograms and summing the reconstructed intensities [2-4]. Digital signal processing methods, which are applied after capture, have also been proposed [5-8].

With respect to three-dimensional (3D) objects, we solve this problem by outof-plane rotating the reference mirror in discrete steps, capturing a hologram each time, and introducing image processing to rectify the resulting shifted diffraction fields. Each rotation amount need not be known and so can be generated by a very fast unstable device. This reference beam rotation is different from [4], where the object beam is rotated at different angles. Changing the angle of incidence of the reference beam changes the phase implicitly captured in the hologram, and results

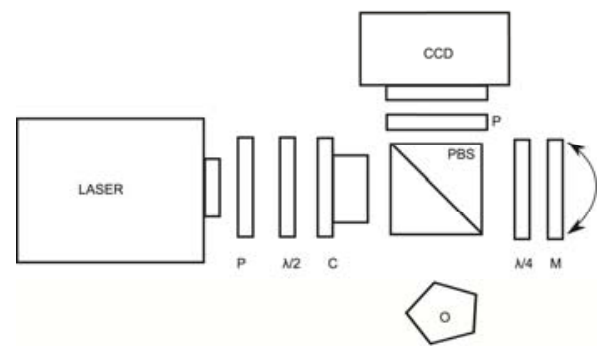

Fig. 1 Experimental Michelson interferometer setup. Polariser (P), half wave plate $(\lambda / 2)$, spatial filter and beam collimator (C), polarising beam splitter (PBS), quarter wave plate $(\lambda / 4)$, mirror $(\mathrm{M})$, object $(\mathrm{O})$. The mirror is rotated between the captures. 
in a different speckle pattern in each reconstructed object. The positions of the object and the camera remain the same so there is no change in perspective between holograms, allowing integration of an arbitrary number of them using the full rotation range allowed by the space-bandwidth product of the camera. The intensities of numerical reconstructions are averaged after image processing registration steps, which produces speckle-reduced reconstructions that closely match theoretical predictions for statistically independent speckle fields.

\section{Method}

Our experimental capturing setup is shown in Fig. 1. The capturing stage is followed by dc and parasitic reflections removal. Each interferogram $H_{k}$ of the set is numerically reconstructed by using a Fresnel intensity approximation given by

$$
I_{k}=\left|\frac{-i}{\lambda z} H_{k}(x, y) * \exp \left[i \pi \frac{\left(x^{2}+y^{2}\right)}{\lambda z}\right]\right|^{2}
$$

where $z$ is the reconstruction distance, $\lambda$ is wavelength of the light, and $*$ denotes a convolution operation. From this set of $\mathrm{n}$ reconstructions $\left\{I_{I}(x, y), I_{2}(x, y), \ldots, I_{n}\right.$ $(x, y)\}$ the first reconstruction is correlated with each of the others in the set which produces a new set of $n-1$ correlation maps, whose maximum peak positions ( shift $_{k}$, shifty ${ }_{k}$ ) for each correlation $k \in[2, n]$ define the image shifts in the horizontal and vertical directions, respectively, required to register the reconstructed objects. Even if the mirror rotation is done only in the horizontal direction, defects or misalignments in the setup may produce need for a vertical shift. By applying these shifts to the reconstructions $\mathrm{I}_{2}(x, y), \ldots, \mathrm{I}_{\mathrm{n}}(x, y)$ and then averaging we obtain

$$
I_{\text {avg }}(x, y)=\frac{1}{n}\left[I_{1}(x, y)+\sum_{k=2}^{n} I_{k}\left(x+\operatorname{shiftx}_{k}, y+\operatorname{shifty}_{k}\right)\right]
$$

In order to reduce the processing time associated with correlation, smaller rescaled versions of the reconstructions are correlated to give coarse estimates of the shifts. According to the approximated $\mathrm{x}$ and $\mathrm{y}$ coordinates of the correlation peaks, the original reconstructions are cropped and correlated again at full resolution but with a small lag in each direction. This final shifts in the $\mathrm{x}$ and $\mathrm{y}$ directions are applied on the original set of intensity reconstructions, which are averaged as explained. Using a personal computer with an Intel Core i7 $1.6 \mathrm{GHz} \mathrm{CPU}, 8 \mathrm{~GB}$ RAM, and interferograms with dimensions $1392 \times 1040$ pixels, the average time needed with this method using a rescale factor of 0.25 (image size becomes $348 \times$ 260 pixels) is 0.42 seconds, a speedup of 7 times compared to not rescaling. 

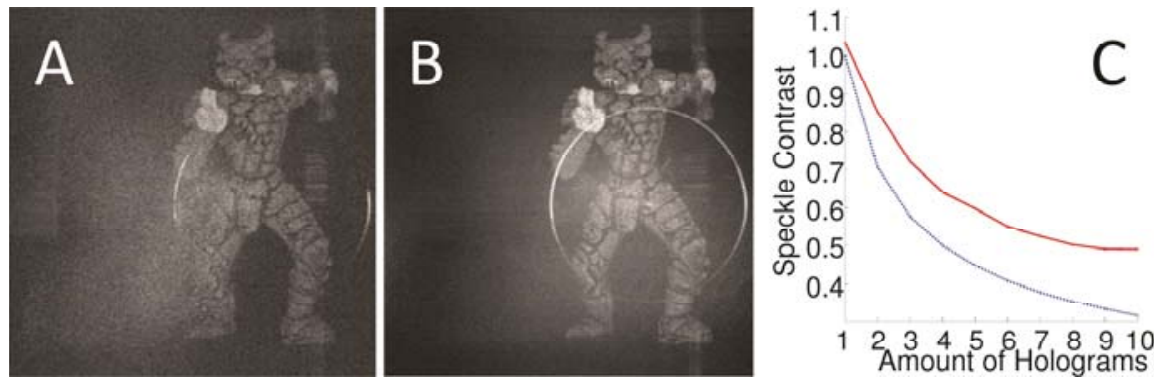

Fig. 2 First reconstruction of the sequence (A) and result from our technique (B). (C) Speckle contrasts with different numbers of reconstructions $n$. Solid line shows the result with our data set with $n=1$ through $n=10$, dotted line is the theoretical speckle reduction as $1 / n^{0.5}$.

\section{Experimental Results}

The experimental results for a $3 \mathrm{~cm}$ tall object are shown in Fig. 2. In the unprocessed intensity reconstructions speckle and unfocussed twin reduce the visibility of the scene and partially blocking the focussed twin. After applying the steps described above the speckle and out of focus twin are significantly reduced. This is verified by calculating a speckle contrast for a constant-intensity region as $\mathrm{c}=\sigma / \Lambda$, where $\Lambda$ is the mean and $\sigma$ is the standard deviation of the speckle intensity. Speckle contrast values were calculated for different numbers of averaged reconstructions, from 1 to 10 . Speckle can be reduced arbitrarily with a quadratic increase in captures and processing time.

The resolution of the procedure was tested with the U.S. Air Force (USAF) 1951 three-bar resolving power test chart. From the Fig. 3B one can see that the resolution remains the same after the procedure.
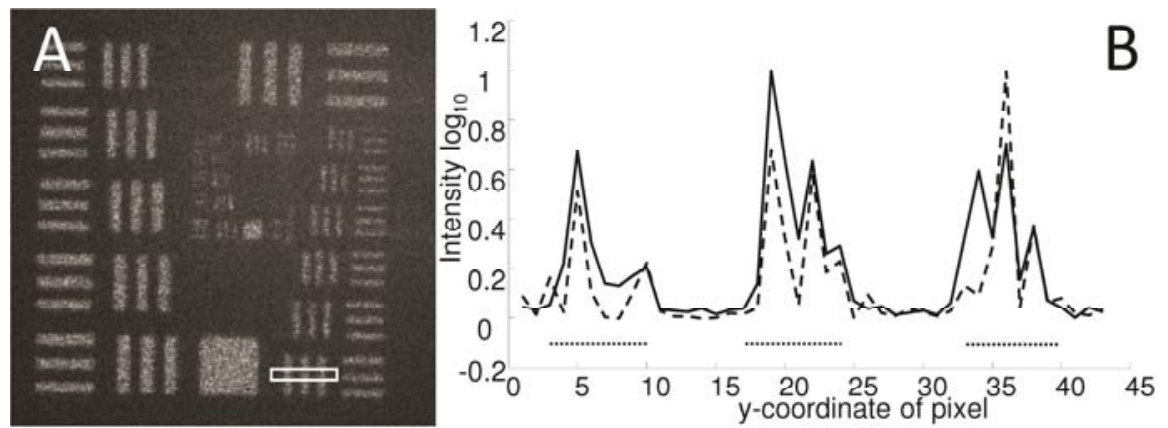

Fig. 3 Resolution. (A) filtered reconstruction with white rectangle showing one pixel row region of interest (ROI), (B) graph showing the resolution of our system. The dashed line is the ROI from the first reconstruction of the set, the solid line is from our averaged reconstruction, and dotted regions at the bottom indicate approximate positions of the bars in the chart. 


\section{Discussion}

Speckle in reconstructions from digital holograms of can be reduced by using rotational mirror at the reference arm of the capturing setup together with image pro-cessing. Experimental results show that this method is able to reduce speckle significantly with real-world 3D objects. The system can be further improved by re-placing the reference mirror in the setup with a liquid crystal on silicon (LCoS) 2D SLM and programming the LCoS to introduce accurate phase tilts. Besides no moving parts, an additional advantage is that reconstructions (or hologram phase in the case of optoelectronic reconstruction) can be spatially registered without a correlation step as the tilt introduced by the LCoS is known accurately. With optoelectronic reconstruction, the SLM could display the phase holograms in rapid succession. If the frame rate is high enough, the statistically-independent speckled reconstructed objects will be averaged by the human retina, which acts as a temporal low-pass filter at high temporal frequencies.

Acknowledgement. This research received funding from the European Community's Seventh Framework Programme FP7/20072013 grant no. 216105 ("Real 3D"), Academy of Finland, and Science Foundation Ireland under the National Development Plan.

\section{References}

1. Goodman, J.W.: Some fundamental properties of speckle. Journal of the Optical Society of America A 66, 1145-1150 (1976)

2. Nomura, T., Okamura, M., Nitanai, E., Numata, E.: Image quality improvement of digital holography by superposition of reconstructed images obtained by multiple wavelengths. Applied Optics 47, D38-D43 (2008)

3. Kelly, D.P., Monaghan, D.S., Pandey, N., Kozacki, T., Michalkiewicz, A., Finke, G., Hennelly, B.M., Kujawinska, M.: Digital holographic capture and optoelectronic reconstruction for 3D displays. International Journal of Digital Multimedia Broadcasting, 114 (2010)

4. Quan, C., Kang, X., Tay, C.J.: Speckle noise reduction in digital holography by multiple holograms. Optical Engineering 46, 115801-115801 (2007)

5. Bertaux, N., Frauel, Y., Réfrégier, P., Javidi, B.: Speckle removal using a maximumlikelihood technique with isoline gray-level regularization. Journal of the Optical Society of America A 21, 2283-2291 (2004)

6. Cai, X.: Reduction of speckle noise in the reconstructed image of digital holography. Optik 121, 394-399 (2010)

7. Memmolo, P., Esnaola, I., Finizio, A., Paturzo, M., Ferraro, P., Tulino, A.M.: SPADEDH: a sparsity-based denoising method of digital holograms without knowing the noise statistics. Optics Express 20, 17250-17257 (2012)

8. Maycock, J., Hennelly, B.M., McDonald, J.B., Frauel, Y., Castro, A., Javidi, B., Naughton, T.J.: Reduction of speckle in digital holography by discrete Fourier filtering. Journal of the Optical Society of America A 24, 1617-1622 (2007) 